\title{
Self-consistent theory of the long-range order in solid solutions
}

\author{
A. I. Olemskoi \\ Department of Physical Electronics, Sumy State University, \\ Rimskii-Korsakov St. 2, 40007 Sumy, Ukrain * $^{*}$
}

(Dated: October 29, 2018)

\begin{abstract}
On the basis of the assumption that atoms play a role of effective Fermions at lattice distribution, the study of the long-range ordering is shown to be reduced to self-consistent consideration of single and collective excitations being relevant to the space distribution of atoms and Fourier transform of such distribution, respectively. A diagram method advanced allows to elaborate complete thermodynamic picture of the long-range ordering of the arbitrary compositional solid solution. The long-range order parameter is found for different chemical potentials of the components to obtain a scope of ordering solid solutions according to relation between degree of the chemical affinity of the components and mixing energy. The boundary composition of the ordering phase $A B_{n}$ is determined as a function of the chemical potentials of the components and concentrations of impurities and defects. Temperature-compositional dependencies of the order parameter and the sublattice difference of the chemical potentials are determined explicitly. Polarization effects and passing out of the compositional domain $0.318<\bar{C}<0.682$ is shown make for transformation of the second order phase transition into the first one. The hydrodynamic behavior of the system is presented by a reactive mode being result of the interference of condensate and fluctuation components of collective excitations. The dispersion law of this mode is displayed experimentally as the Zener peak of the internal friction whose frequency and wave number decay monotonically with temperature increase and phase velocity has a maximum at intermediate temperatures in ordering domain. The polarization effects are shown to be relevant to the static component of Green function, the Goldstone mode of the symmetry restoration is represented by the instant vertex function.
\end{abstract}

PACS numbers: 05.50.+q, 64.60.Cn, 61.66.Dk

\section{INTRODUCTION}

In spite of long history, the problem of long-range order in solid solutions keeps constant interest both of the academic community and for metallurgy applications [1] - 6]. Main peculiarity of contemporary theories along this line consists in using two marginal approaches being based on consideration either of the space distribution of atoms of different kinds or the Fourier transform of such distribution named as concentration waves. In other words, long-range ordering problem is addressed to separate considerations of single or collective excitations distributed over thermodynamic states. However, it is quite clear that above excitations are strong coupled. Thus, the problem appears to study systematically both single and collective excitations in self-consistent manner. That is main purpose of the present paper that is based on the methods proposed initially in quantum statistics [7].

The paper is organized in the following manner. Section II is devoted to statements of used formalism whose key point is that at the lattice sites distribution atoms of a given component is subjected to a prohibition rule to be considered as effective Fermions. This allows us to use the well-known formalism of the second quantization with generic Hamiltonian in a form inherent in the superconductivity theory. In Section III, a diagram method is built up for self-consistent describing both single and collective excitations. This permits to get general equations for the required set of the Green functions. As is shown in Section IV devoted to consideration of the single excitations, their separate description is obtained in the simplest way within the framework of anomalous quasimean value. First, within this approach, we reproduce shortly main results of standard mean-field procedure and then we use the Green function method which allows one to describe not only each of the types of excitations but take into account their coupling as well. Practical advantage of this method is a possibility to study in standard manner solid solutions with arbitrary magnitudes of chemical potential related to both different components and sublattices of ordering structure. As a result, we elaborate complete thermodynamic picture of the longrange ordering of arbitrary solid solution. Consideration of collective excitations in Section $\mathrm{V}$ allows us to reproduce main details of this picture and to take into account polarization effects transforming the second order phase transition into the first one. The hydrodynamic behavior of the system is shown to be presented by a reactive mode being result of the interference of condensate and fluctuation components of collective excitations the last of which is of diffusive type. Concluding Section VI contains a discussion of results obtained.

*Electronic address: blemskoi@ssu.sumy.ua 


\section{EFFECTIVE HAMILTONIAN}

We consider a binary solid solution $A-B$ with components $\mathrm{A}$ and $\mathrm{B}$, which interact with potential $v_{l m}^{a b} \equiv$ $v^{a b}\left(\mathrm{r}_{l}-\mathrm{r}_{m}\right)$ if components $a, b=A, B$ are placed in lattice sites $l, m$ with coordinates $\mathrm{r}_{l}, \mathrm{r}_{m}$, correspondingly. A components distribution over solution sites is given by microscopic occupation numbers $n_{l}^{A}, n_{l}^{B}=0,1$ being obeyed to conservation conditions

$$
\begin{gathered}
n_{l}^{A}+n_{l}^{B}=1, \quad l=1,2, \ldots, N \\
\sum_{l} n_{l}^{a}=N_{a}, \quad a=A, B
\end{gathered}
$$

where $N$ is a total number of the solid solution atoms, $N_{A}, N_{B}$ are the same for components $A, B$ provided $N=$ $N_{A}+N_{B}$. With accounting Eqs. (11), the configurational Hamiltonian of the problem

$$
\mathcal{H}=\frac{1}{2} \sum_{l m} \sum_{a b} v_{l m}^{a b} n_{l}^{a} n_{m}^{b}-\sum_{a l} \mu_{a} n_{l}^{a}
$$

where $\mu_{a}$ is chemical potential of the components $a$, is reduced to usual form

$$
\begin{gathered}
\mathcal{H}=\mathcal{H}_{0}+\mathcal{H}_{1}+\mathcal{H}_{2} ; \\
\mathcal{H}_{0} \equiv \frac{1}{2} \sum_{l m} v_{l m}^{B B}-\mu_{B} N \\
\mathcal{H}_{1} \equiv \sum_{l}\left(\varepsilon_{l}-\mu\right) n_{l}, \quad \varepsilon_{l} \equiv \sum_{m}\left(v_{l m}^{A B}-v_{l m}^{B B}\right) \\
\mu \equiv \mu_{A}-\mu_{B}, \quad n_{l} \equiv n_{l}^{A} \\
\mathcal{H}_{2} \equiv \frac{1}{2} \sum_{l m} w_{l m} n_{l} n_{m}, \quad w_{l m} \equiv v_{l m}^{A A}+v_{l m}^{B B}-2 v_{l m}^{A B}
\end{gathered}
$$

Hereafter, the only $A$-component occupation number $n_{l} \equiv n_{l}^{A}$ will be used.

The corner stone of our approach is that, in sense of the lattice sites distribution, atoms of a given component is subjected to a prohibition rule $n_{l}=0$ or $n_{l}=1$ to be considered as effective Fermions distributed over "states" $l$ [5]. Thus, it is convenient to use the wellknown formalism of the second quantization. With this aim, we represent the occupation numbers in the usual form $n_{l} \equiv a_{l}^{+} a_{l}$ where creation and annihilation operators $a_{l}^{+}, a_{l}$ are subjected to the anticommutation rules $\left\{a_{l}, a_{m}^{+}\right\}=\delta_{l m}$ (as usual, braces denote anticommutator). Moreover, the ordering process causes lattice splitting into two sublattices which will be labelled by Greek indexes 0 or 1 . As a result, Hamiltonian $H \equiv \mathcal{H}-\mathcal{H}_{0}$ counted out the non-essential constant $\mathcal{H}_{0}$ takes the standard second quantization form:

$$
H=\frac{1}{2} \sum_{l \alpha}\left|\varepsilon_{l}-\mu_{\alpha}\right| a_{l \alpha}^{+} a_{l \alpha}+\frac{1}{2} \sum_{l m} w_{l m} a_{m 1}^{+} a_{l 0}^{+} a_{m 0} a_{l 1} .
$$

Here, the anticommutation properties and natural condition $w_{l l} \equiv 0$ (a self-action is absent) take into account, the expressions for a Fermion effective energy $\varepsilon_{l}$, its chemical potential $\mu_{\alpha}$ (being dependent on sublattice number $\alpha$ ) and an effective interaction potential $w_{l m}$ are defined in Eqs. (5), (5). The key point is that atoms $A$ on their own sublattice $\alpha=0$ and on outsider one $\alpha=1$, which chemical potentials are subjected to the condition $\mu_{0}<\varepsilon_{l}<\mu_{1}$, are considered in similar manner as particle and hole in semiconductors. On the other hand, we are kept in last term the only contribution of the sublattices that causes the ordering process $[5]$.

Sometimes, it is convenient to use instead of the above site representation the wave one:

$$
\begin{aligned}
& a_{\mathrm{k}} \equiv \sqrt{\frac{2}{N}} \sum_{l} a_{l} e^{-\mathrm{i} \mathbf{k} \mathbf{r}_{l}}, \quad a_{\mathrm{k}}^{+} \equiv \sqrt{\frac{2}{N}} \sum_{l} a_{l}^{+} e^{\mathrm{i} \mathbf{k} \mathbf{r}_{l}}, \\
& \varepsilon_{\mathbf{k}} \equiv \frac{2}{N} \sum_{l} \varepsilon_{l} e^{\mathrm{i} \mathbf{k} \mathbf{r}_{l}}, \quad w_{\mathbf{k}} \equiv \frac{2}{N} \sum_{l m} w_{l m} e^{\mathrm{i} \mathbf{k}\left(\mathbf{r}_{l}-\mathbf{r}_{m}\right)}
\end{aligned}
$$

where summation is fulfilled over $N / 2$ sites of a sublattice. Then, the expression (7) takes the form

$$
H=\frac{1}{2} \sum_{\mathbf{k} \alpha}\left|\varepsilon-\mu_{\alpha}\right| a_{\mathbf{k} \alpha}^{+} a_{\mathbf{k} \alpha}+\frac{1}{N} \sum_{\mathbf{k k}^{\prime} \mathbf{q}} w_{\mathbf{k}-\mathbf{k}^{\prime}} a_{\mathbf{k} 1}^{+} a_{-\mathbf{k}+\mathbf{q}, 0}^{+} a_{\mathbf{k}^{\prime} 0} a_{-\mathbf{k}^{\prime}+\mathbf{q}, 1} .
$$

Here, we take into account the lattice translational invariance condition according to which the definition (5) derives to a constant Fermion energy $\varepsilon_{l} \equiv \varepsilon$ so that $\varepsilon_{\mathbf{k}}=\varepsilon \delta_{\mathbf{k} 0}$.

\section{DEVELOPMENT OF THE SELF-CONSISTENT SCHEME}

For describing both single and collective excitations, it is convenient to proceed from different (site or wave) representations, so that we shall first make recourse to the diagram method making no use of the explicit form of the appropriate Hamiltonian. This will permit to get the general view of equations for the required set of the Green functions.

Description of the present system is ensured by the use of Matsubara's Green function

$$
G_{l m}^{\alpha \beta}(t)=-\left\langle\widehat{T} a_{l \alpha}(t) a_{m \beta}^{+}(0)\right\rangle
$$

where $a_{l \alpha}^{+}(t), a_{l \alpha}(t)$ are the creation and annihilation operators within the Heisenberg representation, $t$ is the 
imaginary time, the angular brackets mean averaging over configurational states of the solid solution (see [5]), the other symbols are standard [7]. Making use of the above anticommutation relations, it is easy to convince that the normal (diagonal) and anomalous (off-diagonal) components possess the following properties:

$$
\begin{aligned}
& -G_{m l}^{11}(-t)=G_{l m}^{00}(t) \equiv G_{l m}(t), \\
& G_{m l}^{10 *}(t)=G_{l m}^{01}(t) \equiv F_{l m}(t) .
\end{aligned}
$$

Thanks to this, it is sufficient to examine the behavior of only two components - the normal $G_{l m}(t)$ and the anomalous $F_{l m}(t)$ being shown in Figures 1a, b, respectively. Further, when making general computations, it is also convenient to use the matrix representation of the type $\widehat{G}_{l m}(t)$ where the cap signifies the exhaustive search for the $\alpha$ and $\beta$ indices.

FIG. 1: Diagram representation of single and collective excitations

In addition to the matrix nature of the Green function itself, the presence of the sublattices $\alpha, \beta=0,1$ results in matrix structure of the four-tail vertices of the effective interaction depicted in Figure 1c (here, solid line corresponds to a solution component on own sublattice $(\alpha, \beta=0)$ and on another's one $(\alpha, \beta=1)$, the dashed line is respective for the mixture potential $w_{l m}$ ). Since the ordering is caused only by the coupling between components belonging to different sublattices, for fixed indices $l, m$ we shall consider as non-zero only the components $w_{01,01} \equiv w_{00}, w_{10,10} \equiv w_{11}, w_{01,10} \equiv w_{01}$, $w_{10,01} \equiv w_{10}$ producing the $\widehat{w}$ matrix of the second rank (here, one means that interaction $w_{\gamma \delta, \alpha \beta}$ transforms the sublattices $\alpha \beta$ into $\gamma \delta$ ). The $\widehat{\Gamma}$ matrix (see Figure 1d) of the vertex function is structured in the similar manner. However, while the $\widehat{w}$ matrix of the bare interaction is evidently diagonal, the complete vertex $\widehat{\Gamma}$ has, as will be seen below, all nonzero-components.

In the diagram representation the Dyson matrix equation takes an ordinary form depicted in Figure 1e where the thick line corresponds to the exact Green function, and the thin line does to the bare one (the matrix of the latter is diagonal). The self-energy function (Figure 1f) is expressed by the equation illustrated in Figure 1g. As a result, the problem reduces to the self-consistent determination of the vertex function $\widehat{\Gamma}$. It can be shown in standard manner $[7]$ that, at the expansion in terms of $\widehat{w}$, the main contribution is made by the terms containing the polarizer $\widehat{\Pi}$ (see Figure $1 \mathrm{~h}$ ) the matrix components $\Pi_{\alpha \beta}$ of which are determined in a similar way to the $w_{\alpha \beta}$ and $\Gamma_{\alpha \beta}$. Then the appropriate series reduces to the ladder sequence which can be represented in the form of the Bethe-Salpeter equation shown in Figure 1i. It closes the system of equations for the self-consistent description of the ordering process in solid solution.

In the analytic representation, this system is written as follows:

$$
\begin{aligned}
& \widehat{G}^{-1}\left(\omega_{s}\right)=\left[i \omega_{s} \widehat{\delta}+(\widehat{\mu}-\widehat{\varepsilon})\right]-\widehat{\Sigma}\left(\omega_{s}\right), \\
& \Sigma_{\alpha \beta}(t)=G_{\beta \alpha}(-t) \Gamma_{\alpha \beta}(t), \\
& \widehat{\Gamma}^{-1}\left(\Omega_{S}\right)=\widehat{w}^{-1}-\widehat{\Pi}\left(\Omega_{S}\right), \\
& \Pi_{\alpha \beta}(t)=\left[G_{\alpha \beta}(t)\right]^{2} .
\end{aligned}
$$

Here, the frequencies $\omega_{s}=\pi(2 s+1) T, \Omega_{S}=2 \pi S T$ of the single and collective excitations, respectively, are inherent in the Fermi and Bose particles, $T$ is temperature in energy units, $s, S=0, \pm 1, \ldots$ are integers; $\widehat{\delta}$ is a unit matrix, $\widehat{\varepsilon}$ is a diagonal matrix with the elements $\varepsilon^{00}=-\varepsilon^{11}=\varepsilon, \widehat{\mu}$ is the same with the elements $\mu^{00}=\mu_{0}, \mu^{11}=-\mu_{1}\left(\varepsilon, \mu_{\alpha}, \alpha=1,2\right.$ are the bare energy and the sublattice chemical potentials of a Fermion). The matrix structure of the Green function (13) is stated on the Fermi conditions (12). With derivation of the equation (15) for the vertex function $\widehat{\Gamma}$, it is generally taken that the bare potential reduces to the constant $\widehat{w}$. A distinctive feature of the system obtained consists in the fact that the explicit expressions (13), (15) for the Green functions $\widehat{G}, \widehat{\Gamma}$ of the single and collective excitations, respectively, are obtained in the frequency representation, while the expressions (14) and (16) for the self-energy function $\widehat{\Sigma}$ and polarizer $\Pi$ require the application of the time representation. As far as the site and wave representations are concerned, their choice depends on the type of excitations.

\section{SINGLE EXCITATIONS}

If we are interested only in the behavior of the single excitations, their description is obtained in the simplest way within the framework of the quasi-mean value method [8]. Applying the standard procedure, it can be shown on the basis of the expression (7) that in the limit $N \rightarrow \infty$ the behavior of the system is asymptotically defined by the approximating Hamiltonian which takes, in the self-consistent field approximation, the following form:

$\mathcal{H}_{e f} \equiv \frac{1}{2} \sum_{l \alpha}\left|\varepsilon_{l}-\mu_{\alpha}\right| a_{l \alpha}^{+} a_{l \alpha}-\frac{1}{2} \sum_{l}\left(\Delta_{l}^{*} a_{l 0}^{+} a_{l 1}+\Delta_{l} a_{l 1}^{+} a_{l 0}\right)$.

Anomalous quasi-mean values

$$
\eta_{l} \equiv\left\langle a_{l 0}^{+} a_{l 1}\right\rangle, \quad \eta_{l}^{*} \equiv\left\langle a_{l 1}^{+} a_{l 0}\right\rangle
$$

determining the amplitude of the effective Fermion transfer from one sublattice to another represent the (local) order parameter which value gives a gap

$$
\Delta_{l} \equiv-\frac{1}{2} \sum_{m} w_{l m} \eta_{m}, \quad \Delta_{l}^{*} \equiv-\frac{1}{2} \sum_{m} w_{l m} \eta_{m}^{*} .
$$

Here, multipliers $1 / 2$ in front of sums appear to take summation over whole lattice sites (but not over sublattice 
ones). In case of the long-range ordering, the order parameter, given Eq. (18), becomes constant $\left(\eta_{l} \rightarrow \eta\right)$ and, within the usual mean-field approximation, Eqs. (19) give the simple expression for the gap:

$$
\Delta=\frac{W}{2} \eta, \quad W \equiv-\sum_{m} w_{l m} \approx-z w
$$

where the last equality is taken within approximation of interaction of near neighbors which mixing potential is $w$ and number is $z$. For case of the short-range ordering, the correlation parameter (18) slows down with $l$ very fast.

Diagonalization of Hamiltonian (17) is achieved by means of the transformations

$$
\begin{array}{ll}
\alpha_{l+}=u_{l} a_{l 1}^{+}+v_{l} a_{l 0}^{+}, & \alpha_{l-}=u_{l} a_{l 0}-v_{l} a_{l 1} ; \\
a_{l 0}=u_{l} \alpha_{l-}+v_{l} \alpha_{l+}^{+}, & a_{l 1}=u_{l} \alpha_{l+}^{+}-v_{l} \alpha_{l-}
\end{array}
$$

where, by virtue of the anticommutation rule, $u_{l}^{2}+v_{l}^{2}=1$. It results in giving

$$
\mathcal{H}_{e f}=U+\frac{1}{2} \sum_{l} \epsilon_{l}\left(\alpha_{l-}^{+} \alpha_{l-}+\alpha_{l+}^{+} \alpha_{l+}\right)+\frac{1}{2} \sum_{l} \widetilde{\mu}\left(\alpha_{l-}^{+} \alpha_{l-}-\alpha_{l+}^{+} \alpha_{l+}\right)
$$

where we are restricted ourselves with the case of a real order parameter $\left(\eta_{l}=\eta_{l}^{*}\right)$ and one denotes:

$$
\begin{aligned}
& U=\frac{N}{2} \widetilde{\mu}-\frac{1}{2} \sum_{l} \epsilon_{l}, \quad \widetilde{\mu} \equiv \frac{1}{2}\left(\mu_{1}-\mu_{0}\right) ; \\
& \epsilon_{l}=\sqrt{(\bar{\mu}-\varepsilon)^{2}+\Delta_{l}^{2}}, \quad \bar{\mu} \equiv \frac{1}{2}\left(\mu_{0}+\mu_{1}\right) \text {; } \\
& \left.\begin{array}{c}
u_{l}^{2} \\
v_{l}^{2}
\end{array}\right\}=\frac{1}{2}\left(1 \mp \frac{\bar{\mu}-\varepsilon}{\epsilon_{l}}\right) \text {. }
\end{aligned}
$$

The quantity $U$ represents the energy of the ground state whose wave function is

$$
\left|\Psi_{0}\right\rangle=\prod_{l}\left(u_{l}+v_{l} a_{l 1}^{+} a_{l 0}\right)|0\rangle
$$

where $|0\rangle$ is the wave function of the Fermi vacuum. In general case of arbitrary concentration $\bar{C}$ of solid solution, excitations defined by the operators $\alpha_{l+}, \alpha_{l-}$ are non-coincident. Postponing this case below, let us consider at first the simplest case of stoichiometric solution $(\bar{C}=1 / 2)$ where the coincidence of the behavior of elementary excitations in the both states corresponding to sublattices $\alpha=0,1$ is observed $\left(\alpha_{l+} \equiv \alpha_{l-} \equiv \alpha\right)$. It is easy to see that in this case $\widetilde{\mu}=0$.

The equation for the gap is obtained by substitution of the equalities (21) in the definitions (18), (19):

$$
-\frac{1}{2} \sum_{m} w_{l m} \frac{\Delta_{m}}{\epsilon_{m}} \tanh \frac{\epsilon_{m}}{2 T}=\Delta_{l}, \epsilon_{m} \equiv \sqrt{(\bar{\mu}-\varepsilon)^{2}+\Delta_{m}^{2}} .
$$

Here, we have used Fermi distribution for excitations number

$$
\nu_{l} \equiv\left\langle\alpha_{l}^{+} \alpha_{l}\right\rangle=\left[1+\exp \left(\epsilon_{l} / T\right)\right]^{-1}
$$

In the case of the long-range ordering, when dependence of the gap $\Delta_{l} \rightarrow \Delta$ on the site number $l$ disappears, we obtain the usual equation within the mean-field approximation:

$$
\tanh \frac{\epsilon}{2 T}=\frac{2 \epsilon}{W}, \epsilon \equiv \sqrt{(\bar{\mu}-\varepsilon)^{2}+\Delta^{2}}, W \equiv-\sum_{m} w_{l m}
$$

Numerical solution of integral equation (25) allows us to take into account effects of short-range correlations as well, for which the parameter $\Delta_{l}$ is a strong function of the site number $l$.

Concentrations $C_{l \alpha} \equiv\left\langle a_{l \alpha}^{+} a_{l \alpha}\right\rangle$ of $A$-type component on the sublattices $\alpha=0,1$ is determined by Eqs. (21), (23), (26):

$$
\left.\begin{array}{l}
C_{l 0} \\
C_{l 1}
\end{array}\right\}=\frac{1}{2}\left(1 \mp \frac{\bar{\mu}-\varepsilon}{\epsilon_{l}} \tanh \frac{\epsilon_{l}}{2 T}\right)
$$

On the other hand, the difference $\widetilde{\mu}$ of the chemical potentials on the sublattices is given by the condition [9]

$$
\begin{gathered}
\partial \Omega / \partial \widetilde{\mu} \equiv-N \bar{C} \\
\Omega \equiv-T \ln \left\langle\exp \left(-\frac{\mathcal{H}_{e f}-N \tilde{\mu}}{T}\right)\right\rangle, \quad \bar{C} \equiv \frac{1}{2 N} \sum_{l}\left(C_{l 0}+C_{l 1}\right) .
\end{gathered}
$$

Making use of Eqs. (22) gives

$$
\Omega=(U-N \tilde{\mu})+T \sum_{l} \ln \left(1-\nu_{l}\right)
$$

and thermodynamic equalities (29) arrive at trivial re- 
sult $\bar{C}=1 / 2$ for mean concentration. What about the entropy $S \equiv-\partial \Omega / \partial T$, thermodynamic potential (30) derives to usual configuration form

$$
S=-\sum_{l}\left[\nu_{l} \ln \nu_{l}+\left(1-\nu_{l}\right) \ln \left(1-\nu_{l}\right)\right]
$$

With transition to the ordered state, the jump of the thermodynamic potential $\Delta F \equiv F_{\text {ord }}-F_{\text {disord }}$ is determined by the formula

$$
\Delta F=-\frac{1}{2 W} \sum_{l} \Delta_{l}^{2}
$$

following from the equalities $\partial F / \partial \Delta_{l}=\left\langle\partial \mathcal{H}_{e f} / \partial \Delta_{l}\right\rangle$ and (22), (23), (26), (27). According to (32) during ordering, as it usually is during transition to the low-symmetry phase, value $F$ decreases.

The elementary excitation energy $\mathcal{E}_{\text {ex }} \equiv \sum_{l} \epsilon_{l} \nu_{l}$ is at $\bar{C}=1 / 2$ of the form:

$$
\mathcal{E}_{\text {ex }}=\sum_{l} \frac{\sqrt{(\bar{\mu}-\varepsilon)^{2}+\Delta_{l}^{2}}}{1+\exp \left[\sqrt{(\bar{\mu}-\varepsilon)^{2}+\Delta_{l}^{2}} / T\right]} .
$$

Previously, we applied the mean-field method bringing in shortest ways to the description of single excitations. Naturally, the illustrated results can also be obtained by means of the Green function method which allows for determining the Fermi function $\widehat{G}\left(\omega_{s}\right)$ from the system (13) - (16). Keeping in mind the fact that the Green procedure allows for describing not only each of the types of excitations but for taking into account their coupling, we shall demonstrate at first the way it reproduces the results of the mean-field method.

In the context of the above used site representation, the functions $\widehat{G}, \varepsilon$ and $\widehat{\Sigma}$ in the equation (13) and functions $\widehat{\Gamma}, \widehat{w}$ and $\widehat{\Pi}$ in the equation (15) should be corresponded the lattice index $l$, and factorization of the appropriate contribution (14) and (16) is achieved in transition to the wave representation.

To define the explicit form of the Green function (13), we shall consider that, as in the case of the theory of superconductivity, the self-energy function $\widehat{\Sigma}_{l}$ acquires, under ordering, the off-diagonal components $\Sigma_{01 l}=$ $\Sigma_{10 l}^{*}=\Delta_{l}$ corresponding to the gap $\Delta_{l}$ in the energy spectrum of the single excitations $[7]$. The matrix $\widehat{G}_{l}$ inversion leads in the case $\bar{C}=1 / 2$ to the following expressions:

$$
G_{l}\left(\omega_{s}\right)=-\frac{\mathrm{i} \omega_{s}+(\bar{\mu}-\varepsilon)}{\omega_{s}^{2}+\epsilon_{l}^{2}}, \quad F_{l}\left(\omega_{s}\right)=-\frac{\Delta_{l}}{\omega_{s}^{2}+\epsilon_{l}^{2}} ; \quad \epsilon_{l}=\sqrt{(\varepsilon-\bar{\mu})^{2}+\Delta_{l}^{2}}, \quad \omega_{s}=\pi(1+2 s) T, s=0, \pm 1, \ldots
$$

With accounting the sum rules [10]

$$
T \sum_{s=-\infty}^{\infty} \frac{1}{\omega_{s}^{2}+\epsilon^{2}}=\frac{1}{2 \epsilon} \tanh \frac{\epsilon}{2 T}, \sum_{s=-\infty}^{\infty} \frac{\omega_{s}}{\omega_{s}^{2}+\epsilon^{2}}=0
$$

being applicable for some real $\epsilon$, substitution of Eqs. (34) into Eq. (14) for the off-diagonal components, where the vertex $\widehat{\Gamma}_{l}$ is reduced to convolution operator of the bare potential $\widehat{w}_{l} \equiv \sum_{m} w_{l m}$, arrives at the self-consistency equation which, as would be expected, coincides with Eq. (25) for the gap $\Delta_{l}$. It is key point that the diagrammatic approach allows to approve the self-consistency solution of Eq. 25) by means of using some approximation for the vertex $\widehat{\Gamma}_{l}$ (see Section $\mathrm{V}$ ). Dependence of the effective chemical potential $\widetilde{\mu}$ on the difference of the lattice concentrations $C_{l \alpha}$ follows from the relation

$$
\widetilde{C}_{l} \equiv \frac{1}{2}\left(C_{l 1}-C_{l 0}\right)=-T \sum_{s} G_{l}\left(\omega_{s}\right)
$$

which, with the application of the first one from the equalities (34), leads to result

$$
\widetilde{C}_{l}=\frac{\bar{\mu}-\varepsilon}{2 \epsilon_{l}} \tanh \frac{\epsilon_{l}}{2 T}
$$

following from Eq. (28) as well.

Polarizer (16) has the following Fourier-form:

$$
\Pi_{l}^{\alpha \beta}\left(\Omega_{S}\right)=-T \sum_{s=-\infty}^{\infty} \frac{1}{N} \sum_{m} G_{l-m}^{\alpha \beta}\left(\Omega_{S}-\omega_{s}\right) G_{m}^{\alpha \beta}\left(\omega_{s}\right) .
$$

Substituting the Green functions (34) here, upon summing over $s$, we arrive at the expressions 


$$
\begin{gathered}
\Pi^{00}(0)=\Pi^{11}(0)=-\frac{1}{2 N} \sum_{l} \epsilon_{l}^{-1} \tanh \frac{\epsilon_{l}}{2 T}+A \Delta^{2}, \quad \Pi^{01}(0)=\Pi^{10}(0)=-A \Delta^{2} ; \\
A \equiv \frac{1}{4 N} \sum_{l} \epsilon_{l}^{-3}\left(\tanh \frac{\epsilon_{l}}{2 T}-\frac{\epsilon_{l}}{2 T} \cosh ^{-2} \frac{\epsilon_{l}}{2 T}\right),
\end{gathered}
$$

where, in view of the macroscopic equivalence of sites, there is no dependence on their number $l$. Making use of Eq. (38), we find the inverse vertex function (15) responsible for the behavior of the collective excitations (see Section $\mathrm{V}$ ).

The system of equations (18), (19), (25), (29) and (30) that offers the self-consistent description of the single excitations affords finding the gap $\Delta$, the order parameter $\eta$ and the sublattice difference of concentrations $\widetilde{C}$ by the assigned values of thermodynamic parameters (the temperature $T$ and the chemical potential $\bar{\mu}$ ), as well as microscopic parameters (the value of the mixing potential $w_{l m}$ and effective energy $\varepsilon$ given by Eqs. (5), (5)). Numerical solution of these equations for the long-range order parameter results in the temperature dependencies displayed in Figure 2. From this figure we notice that

FIG. 2: Temperature dependencies of the long-range order parameter at different magnitudes of chemical potentials (curves top-down correspond to values $(\bar{\mu}-\varepsilon) / \Delta_{0}=$ $0.0,0.2,0.3,0.4,0.5,0.6,0.7,0.8,0.9$. Concentration $\bar{C}=0.5)$

the parameter $\eta$ monotonically decays as the temperature $T$ and the difference $|\bar{\mu}-\varepsilon|$ increase. According to Eqs. (27), (37), at long-range ordering the sublattice concentration difference is

$$
\widetilde{C}=\frac{\bar{\mu}-\varepsilon}{W}
$$

to be determined by the mean chemical potential but being non-dependent on temperature. Usually, one supposes $\bar{\mu}=\varepsilon$ and, as consequence, $\widetilde{C}=0$ (i.e., $C_{0}=C_{1}$ ).

The ground system state defined by the function (24) is achieved at $T=0$ where site dependence is missing and excitations are absent $(\nu=0)$. At $\bar{\mu}=\varepsilon$ the gap width and order parameter take maximum values $\Delta_{0}=\frac{1}{2} W \equiv-\frac{1}{2} \sum_{m} w_{l m}, \eta=1$, variation of the ground state energy, being equal to the decreasing thermodynamic potential, takes maximum value $|\Delta F|=\frac{N}{4} \Delta_{0}$; respectively, the sublattice concentrations $C_{0}, C_{1}$ coincide. With finite difference $\bar{\mu}-\varepsilon \neq 0$, there are $\Delta=\sqrt{\Delta_{0}^{2}-(\bar{\mu}-\varepsilon)^{2}}, \eta=\sqrt{1-(\bar{\mu}-\varepsilon)^{2} / \Delta_{0}^{2}}, \Delta F=$ $-\frac{N}{4} \Delta_{0}\left[1-(\bar{\mu}-\varepsilon)^{2} / \Delta_{0}^{2}\right], \widetilde{C}=(\bar{\mu}-\varepsilon) / 2 \Delta_{0}$. Thus, as the sublattice chemical potentials $\mu_{0}=\mu_{1}=\bar{\mu}$ increases from value $\varepsilon$ to $\varepsilon+\Delta_{0}$, the gap width, order parameter and absolute value of the system free energy change monotonically decay going into zero at the boundary value $\bar{\mu}_{c}=\varepsilon+\Delta_{0}$. The sublattice concentrations difference varies here linearly from 0 to $1 / 2$.

As the temperature arises, originated are the elementary excitations the number of which $\nu \approx$ $\exp \left(-\Delta_{0} / T\right) \ll 1$. With a precision of the first power terms over $\nu \ll 1$, we obtain:

$$
\begin{gathered}
\Delta=\Delta_{0} \sqrt{1-\left(\frac{\bar{\mu}-\varepsilon}{\Delta_{0}}\right)^{2}}\left\{1-2\left[1-\left(\frac{\bar{\mu}-\varepsilon}{\Delta_{0}}\right)^{2}\right]^{-1} \exp \left(-\frac{\Delta_{0}}{T}\right)\right\}, \\
\eta=\frac{\Delta}{\Delta_{0}}, \quad \widetilde{C}=\frac{\bar{\mu}-\varepsilon}{2 \Delta_{0}}, \quad \Delta_{0}=\frac{1}{2} W \equiv-\frac{1}{2} \sum_{m} w_{l m}, \\
\Delta F=-\frac{N}{4} \Delta_{0} \eta^{2}, \quad \mathcal{E}_{\text {ex }}=N \Delta_{0} \exp \left(-\frac{\Delta_{0}}{T}\right) .
\end{gathered}
$$

As it is known, the exponential character is inherent in the low-temperature dependencies usually observed under phase transitions.

Near critical temperature $T_{c}=W / 4$ the ordering process can be presented in analytical form within the mean-field approximation that is stated on making use of Eq. (27). Within domain $0<\left(T_{c}-T\right) / T_{c} \ll 1$, we obtain:

$$
\begin{gathered}
\Delta=2 T_{c} \sqrt{3 t}, \quad \eta=\sqrt{3 t}, \quad t \equiv \frac{T_{c}-T}{T_{c}}, \quad T_{c}=\frac{1}{4} W, \quad W \equiv-\sum_{m} w_{l m}, \\
\Delta F=-\frac{3}{2} N T_{c} t, \quad \mathcal{E}_{\mathrm{ex}}=N T_{c} \sqrt{3 t}
\end{gathered}
$$

where we put $\bar{\mu}=\varepsilon$. 
Now, we consider general case of non-stoichiometric solid solution which the (mean) concentration $\bar{C} \neq 1 / 2$ and the difference $\tilde{\mu}$ of the sublattice chemical potentials is determined by equations (cf. Eq. (29))

$$
\begin{gathered}
\partial \Omega / \partial \widetilde{\mu} \equiv-N \bar{C} ; \\
\Omega=-\frac{N}{2} \widetilde{\mu}-\frac{1}{2} \sum_{l} \epsilon_{l}+T \sum_{l} \ln \left(1-\nu_{l+}\right)+T \sum_{l} \ln \left(1-\nu_{l-}\right), \\
\nu_{l \pm}=\left\{1+\exp \left(\frac{\epsilon_{l} \mp \widetilde{\mu}}{2 T}\right)\right\}^{-1}
\end{gathered}
$$

following from Eqs. (29), (22) and (23). Respectively, inversion of matrix (13) gives instead of Eqs. (34)

$$
\begin{gathered}
G_{l}^{00}\left(\omega_{s}\right)=\left[\mathrm{i} \omega_{s}-\left(\mu_{1}-\varepsilon\right)\right] / \mathcal{D}_{l}, \quad G_{l}^{11}\left(\omega_{s}\right)=\left[\mathrm{i} \omega_{s}+\left(\mu_{0}-\varepsilon\right)\right] / \mathcal{D}_{l}, \\
G_{l}^{01}\left(\omega_{s}\right)=\Delta_{l} / \mathcal{D}_{l}, \quad G_{l}^{10}\left(\omega_{s}\right)=\Delta_{l}^{*} / \mathcal{D}_{l} ; \quad \mathcal{D}_{l}\left(\omega_{s}\right) \equiv\left[\mathrm{i} \omega_{s}-\left(\epsilon_{l}+\widetilde{\mu}\right)\right]\left[\mathrm{i} \omega_{s}+\left(\epsilon_{l}-\widetilde{\mu}\right)\right], \\
\epsilon_{l}=\sqrt{(\bar{\mu}-\varepsilon)^{2}+\left|\Delta_{l}\right|^{2}}, \quad \bar{\mu} \equiv \frac{1}{2}\left(\mu_{0}+\mu_{1}\right), \quad \widetilde{\mu} \equiv \frac{1}{2}\left(\mu_{1}-\mu_{0}\right), \\
\omega_{s}=\pi(1+2 s) T, \quad s=0, \pm 1, \ldots
\end{gathered}
$$

With accounting Eqs. (35) and Eq. (14) with $\widehat{\Gamma}$ substituted by $\widehat{w}$, we obtain the self-consistency condition

$$
-\frac{1}{4} \sum_{m} w_{l m} \frac{\Delta_{m}}{\epsilon_{m}}\left(\tanh \frac{\epsilon_{m}+\tilde{\mu}}{2 T}+\tanh \frac{\epsilon_{m}-\tilde{\mu}}{2 T}\right)=\Delta_{l}
$$

replacing Eq. (25). Respectively, from equalities $C_{\alpha} \equiv-T \sum_{s} G_{\alpha \alpha}\left(\omega_{s}\right)$ one follows:

$$
\begin{aligned}
C_{0} & =\frac{\epsilon-(\bar{\mu}-\varepsilon)}{4 \epsilon} \tanh \frac{\epsilon+\widetilde{\mu}}{2 T}-\frac{\epsilon+(\bar{\mu}-\varepsilon)}{4 \epsilon} \tanh \frac{\epsilon-\widetilde{\mu}}{2 T}, \\
C_{1} & =\frac{\epsilon+(\bar{\mu}-\varepsilon)}{4 \epsilon} \tanh \frac{\epsilon+\widetilde{\mu}}{2 T}-\frac{\epsilon-(\bar{\mu}-\varepsilon)}{4 \epsilon} \tanh \frac{\epsilon-\tilde{\mu}}{2 T} .
\end{aligned}
$$

In case of the long-range ordering, when the parameters $\Delta_{l} \rightarrow \Delta, \epsilon_{l} \rightarrow \epsilon$ do not depend on the site number $l$, the relations (45), 46 arrive at Eq. (40), as follows. On the other hand, thermodynamic equality (43) derive to equation

$$
2 \bar{C}-1=\frac{1}{N} \sum_{l}\left(\nu_{l+}-\nu_{l-}\right) \equiv \frac{1}{2 N} \sum_{l}\left(\tanh \frac{\epsilon_{l}+\widetilde{\mu}}{2 T}-\tanh \frac{\epsilon_{l}-\tilde{\mu}}{2 T}\right)
$$

where the relation $1-2 \nu_{l \pm}=\tanh \frac{\epsilon_{l} \mp \widetilde{\mu}}{2 T}$ is taken into account. In difference of Eq. (29), this equation is non-trivial and accompanied with Eqs. (45), (46) determines quantities $\Delta_{l}, C_{0}, C_{1}, \widetilde{\mu}$ at given thermodynamic parameters $T$, $\bar{C}, \bar{\mu}$ and microscopic ones $w_{l m}$ and $\varepsilon$. In similar manner, the excitation energy is determined by the expressions (cf. Eq. (33))

$$
\mathcal{E}_{\mathrm{ex}} \equiv \sum_{l} \epsilon_{l}\left(\nu_{l+}+\nu_{l-}\right)=\Delta_{0} \sum_{l}\left\{1+\frac{\cosh \left(\epsilon_{l} / 2 T\right)}{\cosh (\widetilde{\mu} / 2 T)}\right\}^{-1}
$$

at $\bar{\mu}=\varepsilon$. The entropy $S \equiv-\partial \Omega / \partial T$ is reduces to the configuration form type of Eq. (31):

$$
S=-\sum_{l}\left\{\left[\nu_{l+} \ln \nu_{l+}+\left(1-\nu_{l+}\right) \ln \left(1-\nu_{l+}\right)\right]+\left[\nu_{l-} \ln \nu_{l-}+\left(1-\nu_{l-}\right) \ln \left(1-\nu_{l-}\right)\right]\right\} .
$$

In the simplest case $\bar{\mu}=\varepsilon$ equations (45), (47) govern the long-range ordering to be reduced to the form

$$
\begin{aligned}
& \tanh \frac{\eta+\widetilde{m}}{\Theta}+\tanh \frac{\eta-\tilde{m}}{\Theta}=2 \eta, \\
& \tanh \frac{\eta+\widetilde{m}}{2 \Theta}-\tanh \frac{\eta-\tilde{m}}{2 \Theta}=2(2 \bar{C}-1)
\end{aligned}
$$

where dimensionless values of the sublattice difference of the chemical potentials $\widetilde{m} \equiv \widetilde{\mu} / \Delta_{0}$ and temperature $\Theta \equiv$ 
$T / T_{c}$ are introduced. Analytical consideration of this system is possible in the limits $\Theta \ll 1$ and $\eta \ll 1$ only. In the first case, with accuracy of the first non-vanished terms, equations (50), (51) arrive at the expressions

$$
\begin{gathered}
\eta=\eta_{0}-\exp \left(-\frac{\Delta_{0} \eta_{0}}{T}\right), \\
\widetilde{\mu}=\Delta_{0} \eta_{0}+\alpha T
\end{gathered}
$$

where parameters $\eta_{0}, \alpha$ are determined by relations

$$
\eta_{0}=\frac{1}{2}\left(1-\tanh \frac{\alpha}{2}\right)=\frac{(1-\bar{C})^{2}}{\frac{1}{8}+2\left(\bar{C}-\frac{3}{4}\right)^{2}} .
$$

The phase transition curve where

$$
\eta=0, \cosh \frac{\widetilde{m}}{\Theta}=\frac{1}{\sqrt{\Theta}}=\frac{1+\delta^{2}}{1-\delta^{2}} ; \quad \delta \equiv 2 \bar{C}-1
$$

is determined by relations

$$
2 \bar{C}-1=\frac{\sqrt{T_{c}-T}}{\sqrt{T_{c}}+\sqrt{T}}, \quad T=T_{c}\left(\frac{1-\delta^{2}}{1+\delta^{2}}\right)^{2} .
$$

In the vicinity of the transition curve $T_{c}(\delta)$ one has

$$
\begin{gathered}
\eta^{2}=A(\delta) t(\delta), \quad t(\delta) \equiv \frac{T_{c}(\delta)-T}{T_{c}(\delta)} \\
\cosh \frac{\widetilde{\mu}}{2 T}=\frac{1+\delta^{2}}{1-\delta^{2}}+\frac{A(\delta) \delta^{2}}{1-\delta^{2}} t(\delta)
\end{gathered}
$$

where factor $A(\delta)$ is determined by the equality

$$
A^{-1} \equiv \frac{2 \delta^{2}}{1+\delta^{2}}+\left[\left(\frac{1-\delta^{2}}{1+\delta^{2}}\right)^{2}-\frac{2}{3}\right]\left(\frac{1+\delta^{2}}{1-\delta^{2}}\right)^{4} .
$$

With deviation of the stoichiometric composition this factor decays as $A \simeq \sqrt{3}\left(1-2 \delta^{2}\right), \delta^{2} \ll 1$ taking zeroth magnitude at $\delta_{0}=0.364\left(C_{0}=0.682\right)$. Physically, this means that with passing out of the domain $0.318<\bar{C}<0.682$ the second order phase transition is transformed into the first one being close to the second.

FIG. 3: Temperature dependencies of the long-range order parameter (a) and the sublattice difference of the chemical potentials (b) at different concentrations $\bar{C}$ being pointed out near corresponding curves. (The dashed line bounds the abruption region)

Explicit forms of the dependencies $\eta(T, \bar{C}), \widetilde{\mu}(T, \bar{C})$ in the case $\bar{\mu}-\varepsilon=0$ are depicted in Figures 3, 4. The order parameter $\eta$ decays monotonically with increase of both temperature and concentration (see Figures 3a, $4 \mathrm{a}$ ), whereas behavior of the sublattice difference of the chemical potentials $\widetilde{\mu}$ is much more complicated: when the temperature grows, it decreases near the stoichiometric composition $\bar{C}=0.5$ passing to increasing regime with approaching to the composition $\bar{C}=1$ (Figure $3 \mathrm{~b}$ ); analogous behavior is observed with deviation of the stoichiometric composition at low and pre-critical temperatures (Figure 4b). Principle important is the interruption
FIG. 4: Compositional dependencies of the long-range order parameter (a) and the sublattice difference of the chemical potentials (b) at different temperatures (the magnitudes of the latter related to critical value are pointed out near corresponding curves). The dashed line bounds the abruption region

of the sublattice difference of the chemical potentials $\widetilde{\mu}$ at the curve of the phase transition that takes maximal values within intermediate ordering region.

If the system is subjected to external influence, the parameter $\bar{m} \equiv(\bar{\mu}-\varepsilon) / \Delta_{0}$ becomes non-zeroth and ordering process is described by the system (50), (51) where the order parameter $\eta$ should be replaced by the renormalized one $\sqrt{\eta^{2}+\bar{m}^{2}}$. It is easy to convince oneself the growth of the influence parameter $\bar{m}$ causes suppressing ordering process to shrink the long-range domain as shown in Figure 5a. At that, the boundary composition being the solubility limit of the ordering phase is determined by the parameter of external influence in following manner:

$$
\bar{C}=1-\frac{\sqrt{\bar{m}(1-\bar{m})}-\bar{m}}{2(1-2 \bar{m})}, \quad \bar{m} \equiv \frac{\bar{\mu}-\varepsilon}{\Delta_{0}} .
$$

According to related plot in Figure $5 \mathrm{~b}$ the solubility concentration decays monotonically with arising external influence to vary anomalously fast near boundary magnitudes $\bar{C}=1$ and $\bar{C}=0.5$.

FIG. 5: a - The phase diagram determining long-range order (LRO) and disorder (DO) domains at different magnitudes of chemical potentials (curves top-down correspond to values $\left.(\bar{\mu}-\varepsilon) / \Delta_{0}=0.0,0.2,0.3,0.4,0.5,0.6,0.7,0.8,0.9\right) . \mathrm{b}-$ The solubility limit of the ordering phase versus the parameter of external influence

\section{COLLECTIVE EXCITATIONS}

As is seen from Sections II and IV, the ordering process results from the fact that, as the temperature decreases, the effective Fermions form coupled pairs corresponding to the collective excitations of the Bose type. If the number of such pairs $\mathcal{N}=\frac{1}{2} N \eta$ makes up the finite part with respect to the total number of sites $N$, the ordering process assumes the macroscopic character and is determined by the long-range order parameter $\eta=\Delta / \Delta_{0}$. So, in the representation of single excitations, the solid solution ordering is reflected through the appearance of the off-diagonal components $\Delta$ in the matrix of the selfenergy function $\widehat{\Sigma}$.

Let us show now how the ordering manifests itself in the representation of the collective excitations. Application of the mean-field method, therewith, turns to be insufficient and a recourse should be made to the selfconsistent scheme developed in Section III to take into 
account the behavior of the Green functions of both single and collective excitations. To study the latter we start with consideration of the Fourier representation $\widehat{\Gamma}(\mathbf{K}, \Omega)$ of the vertex function acquiring under ordering a condensate component $\widehat{\Gamma}_{0}(\mathbf{K}, \Omega)$ accompanied by a fluctuation function $\widehat{\Gamma}^{\prime}(\mathbf{K}, \Omega)$. Their principle difference is in hydrodynamic behavior in the limit $\mathbf{K}, \Omega \rightarrow 0$ where the latter tends to zero, whereas the former takes a finite magnitude $\widehat{\Gamma}_{0} \neq 0$. For determination of the latter we substitute the polarizer (38) into (15):

$$
\widehat{\Gamma}_{0}^{-1}=\left(\begin{array}{cc}
B-A|\Delta|^{2} & A \Delta^{2} \\
A\left(\Delta^{*}\right)^{2} & B-A|\Delta|^{2}
\end{array}\right), \quad B \equiv-W^{-1}+\frac{1}{2 \epsilon} \tanh \frac{\epsilon}{2 T}
$$

where account is taken of the diagonal structure of the interaction matrix $\widehat{w}$, the parameter $A$ is determined by the last equality (39) (in this Section, the gap $\Delta$ is supposed to be complex). It is principally important the parameter $B \rightarrow 0$ with ordering, in accordance with condition Eq. (25) for the gap $|\Delta|$.

Inversion of the matrix (61) arrives at the off-diagonal component of the condensate vertex:

$$
\Gamma_{0}^{01}=-\Delta_{0} \frac{\frac{1}{\eta} \tanh \frac{\eta}{\Theta}-\frac{1}{\Theta} \cosh ^{-2} \frac{\eta}{\Theta}}{\left(1-\frac{1}{\eta} \tanh \frac{\eta}{\Theta}\right)\left(1-\frac{1}{\Theta} \cosh ^{-2} \frac{\eta}{\Theta}\right)} .
$$

Near the critical point $\left(T-T_{c} \ll T_{c}\right)$ where

$$
\Gamma_{0}^{01} \simeq-\frac{2}{3} \Delta_{0} \frac{\eta^{2}}{\left(1-T / T_{c}\right)^{2}}
$$

we can neglect a frequency dispersion to put $\Sigma^{01}(\Omega) \simeq$ $\Sigma^{01}(0), \Gamma^{01}(\Omega) \simeq \Gamma^{01}(0) \equiv \Gamma_{0}^{01}$ in Fourier transform of the equality (14) that takes the form

$$
\frac{1}{\eta} \tanh \frac{\eta}{\Theta}=-2 \frac{\Delta_{0}}{\Gamma_{0}^{01}} .
$$

As a result, the collective excitations are characterized by the relations

$$
\eta \simeq \sqrt{3}\left(1-\frac{T}{T_{c}}\right), \Gamma_{0}^{01} \simeq-2 \Delta_{0} ; \quad T-T_{c} \ll T_{c}
$$

the first of which is principally different of the corner peculiarity (57) inherent in the single excitations. This difference is expressed physically in that the collective excitations polarize ordering system to transform the second order phase transition into the first one.

Now, we are in position to study the collective excitations themselves. Their Green function is determined by the fluctuation component $\widehat{\Gamma}^{\prime}(\mathbf{K}, \Omega)$ of the vertex function according to the relation

$$
\widehat{\varphi}^{\prime}(\mathbf{K}, \Omega) \equiv-\frac{1}{2 \pi \mathrm{i}} \widehat{\Gamma}^{\prime}(\mathbf{K}, \Omega)
$$

The simplest way to find this function in hydrodynamic limit $\mathbf{K}, \Omega \rightarrow 0$ is to apply the method developed in Refs. [11], 12]. Its main point consists in the fact that instead of the equation $\widehat{G}^{-1}=\widehat{G}_{0}^{-1}-\Delta_{0} \widehat{\delta}$, for the Green function $\widehat{G}$, use is made of its analog $\langle\widehat{G}\rangle^{-1}=$ $\widehat{\sigma}^{-1}-\Delta_{0} \widehat{\delta}$ for the averaged function $\langle\widehat{G}\rangle$ and locator $\widehat{\sigma}=\left\langle\widehat{G}_{0}\right\rangle$. In addition, inserted is the effective interactor $\widehat{U}=\Delta_{0} \widehat{\delta}+\Delta_{0}^{2}\langle\widehat{G}\rangle$ which contains the term in the first order over Fermion energy. The Dyson equation replacing (13) therewith assumes the form $\widehat{U}^{-1}=\Delta_{0}^{-1} \widehat{\delta}-\widehat{\sigma}$. The two-particle Green function

$$
\varphi(E ; \mathbf{K}, \Omega)=-\frac{1}{2 \pi \mathrm{i}} \sum_{\mathbf{k}, \mathbf{k}^{\prime}}\left\langle G^{\mathrm{R}}\left(\mathbf{k}_{+}, \mathbf{k}_{+}^{\prime} ; E+\Omega\right) G^{\mathrm{A}}\left(\mathbf{k}_{-}, \mathbf{k}_{-}^{\prime} ; E\right)\right\rangle
$$

appears, in the ladder approximation, as (compare with (15p)

$$
\varphi(E ; \mathbf{K}, \Omega)=-\frac{1}{2 \pi \mathrm{i}}\left[\gamma^{-1}-\sum_{\mathbf{k}} U^{\mathrm{R}}\left(\mathbf{k}_{+} ; E+\Omega\right) U^{\mathrm{A}}\left(\mathbf{k}_{-} ; E\right)\right]^{-1}
$$

where $\mathbf{k}_{ \pm}=\mathbf{k} \pm \mathbf{K} / 2, \gamma$ is an irreducible four-tail vertex, indices $\mathrm{R}$ and $\mathrm{A}$ of the retarded and advanced functions correspond to the selection of the components $\alpha=0,1$ in various sublattices. Hence, taking into account the Dyson equation and Ward identity [7], we have

$$
\sigma_{\mathbf{k}_{+}}^{\mathrm{R}}(E+\Omega)-\sigma_{\mathbf{k}_{-}}^{\mathrm{A}}(E)=\gamma \sum_{\mathbf{k}^{\prime}}\left[U^{\mathrm{R}}\left(\mathbf{k}_{+}^{\prime} ; E+\Omega\right)-U^{\mathrm{A}}\left(\mathbf{k}_{-}^{\prime} ; E\right)\right]-\widetilde{\gamma} \Omega
$$


where the irreducible vertex $\widetilde{\gamma}$ has, contrary to the $\gamma$, two coinciding tails appropriate to the same sites. Then, we come to the conventional expression for the fluctuation component of the two-particle Green function

$$
\varphi^{\prime}(\mathbf{K}, \Omega)=-\frac{\chi(\mathbf{K})}{\Omega+\mathrm{i} D(\mathbf{K}, \Omega) \mathbf{K}^{2}}
$$

where $\chi(\mathbf{K})$ is susceptibility taking in hydrodynamic limit $\mathbf{K} \rightarrow 0$ the thermodynamic value $\chi, D(\mathbf{K}, \Omega)$ is the dispersing diffusion coefficient. As a result of the above, for the fluctuation component of the vertex function we obtain 11, 12

$$
\left(\widehat{\Gamma}^{\prime}(\mathbf{K}, \Omega)\right)^{-1}=\frac{1}{2 \pi \chi}\left(\begin{array}{cc}
-\mathrm{i} \Omega+D \mathbf{K}^{2} & 0 \\
0 & \mathrm{i} \Omega+D \mathbf{K}^{2}
\end{array}\right)
$$

where the matrix structure reflects the presence of two poles $\Omega=\mp \mathrm{i} D \mathbf{K}^{2}$.

The sum of the expression (61) with $B=0$ and Eq. (71) produces, after the matrix inversion, the complete Green function of collective excitations:

$$
\begin{gathered}
\widehat{\varphi}(\mathbf{K}, \Omega)=\frac{\chi}{\mathcal{D}(\mathbf{K}, \Omega)}\left(\begin{array}{cc}
-\Omega-\mathrm{i}\left(\frac{1}{2} S K_{0}-D \mathbf{K}^{2}\right) & -\frac{\mathrm{i}}{2}(\Delta /|\Delta|)^{2} S K_{0} \\
-\frac{\mathrm{i}}{2}\left(\Delta^{*} /|\Delta|\right)^{2} S K_{0} & \Omega-\mathrm{i}\left(\frac{1}{2} S K_{0}-D \mathbf{K}^{2}\right)
\end{array}\right), \\
\mathcal{D}(\mathbf{K}, \Omega)=\Omega^{2}+\left((1 / 2) S K_{0}-D \mathbf{K}^{2}\right)^{2}-(1 / 4) S^{2} K_{0}^{2}, \\
S^{2}=4 \pi \chi A|\Delta|^{2} D, \quad K_{0}^{2}=4 \pi \chi A|\Delta|^{2} / D .
\end{gathered}
$$

Condition $\mathcal{D}(\mathbf{K}, \Omega)=0$ brings to the dispersion law

$$
\Omega=\mp D K \sqrt{K_{0}^{2}-\mathbf{K}^{2}}
$$

whose characteristic feature consists in a bell form with a slanting long-wave side (see Figure 6). Physically, the dependence (75) relates to the collective mode that is of a reactive nature in the long-wave region $K<K_{0}$ limited by the wave number given Eq. (74) and has a relaxation nature in the short-wave region $K>K_{0}$. At $K \gg K_{0}$, it transforms into the ordinary diffusion mode $\Omega=\mp \mathrm{i} D \mathbf{K}^{2}$.

FIG. 6: Law of collective mode dispersion (the solid line corresponds to the real value of frequency $\Omega$, the dashed - imaginary value, the dotted - diffusion mode)

In this way, the self-consistent consideration of single and collective excitations leads to the conclusion that apart from the diffusion regime realized in the mesoscopic region $K>K_{0}$, possible in the system is quasioscillations characterized by the phase velocity $S$ determined by the first of equalities (74). Obviously, the dispersion law (75) acquiring the acoustic form in the limiting long-wave region $K \ll K_{0}$ is observed as Zener peak in experiments with internal friction [13]. This mode is characterized by the frequency

$$
\Omega_{0} \equiv S K_{0}=\frac{\pi \chi}{\Delta_{0}}\left(\frac{1}{\eta} \tanh \frac{\eta}{\Theta}-\frac{1}{\Theta} \cosh ^{-2} \frac{\eta}{\Theta}\right)
$$

whose temperature dependence at concentration $\bar{C}=0.5$ is shown in Figure 7 with solid line. This frequency is seen to decay monotonically from the magnitude $\Omega_{0}=\pi \chi / \Delta_{0}$ at $T=0$ to $\Omega_{0}=0$ at $T=T_{c}$ varying near the critical point quadratically:

$$
\Omega_{0} \simeq 2 \pi \frac{\chi}{\Delta_{0}}\left(1-\frac{T}{T_{c}}\right)^{2}, \quad T-T_{c} \ll T_{c} .
$$

Here, we take into account polarization effects transforming the corner peculiarity (57) into the linear relation (65). Since the diffusion coefficient $D=$

FIG. 7: Temperature dependencies of the characteristic frequency $\Omega_{0}$ (solid line), phase velocity $S$ (broken line) and boundary value of the wave number $K_{0}$ (dotted line). Magnitudes $\Omega_{0}, S$ and $K_{0}$ are measured in units $\pi \chi / \Delta_{0}$, $\sqrt{\pi \chi D_{0} / \Delta_{0}}$ and $\sqrt{\pi \chi / \Delta_{0} D_{0}}$, respectively. The height of diffusion barrier is put to be $Q=3 T_{c}$. The insertion is built with accounting polarization effects given by Eqs. (77) - (79)

$D_{0} \exp \{-(Q / T)\}$ is determined by the energy barrier $Q$ to behave itself in a non-critical manner, the parabolic dependence (77) means the linear behavior for both the sound velocity and the boundary wave number:

$$
\begin{aligned}
& S \simeq \sqrt{2 \pi \frac{\chi D_{0}}{\Delta_{0}}} \exp \left(-\frac{Q}{2 T_{c}}\right)\left(1-\frac{T}{T_{c}}\right), \\
& K_{0} \simeq \sqrt{2 \pi \frac{\chi}{\Delta_{0} D_{0}}} \exp \left(\frac{Q}{2 T_{c}}\right)\left(1-\frac{T}{T_{c}}\right)
\end{aligned}
$$

the former of which is exponentially smaller than the latter. With the temperature falling down in the domain $T \ll T_{c}$ the sound velocity decreases and the boundary value of the wave number, on the contrary, infinitely in- 
creases:

$$
\begin{aligned}
& S \simeq \sqrt{\frac{\pi \chi D_{0}}{\Delta_{0}}} \exp \left(-\frac{Q}{2 T}\right), \\
& K_{0} \simeq \sqrt{\frac{\pi \chi}{\Delta_{0} D_{0}}} \exp \left(\frac{Q}{2 T}\right) .
\end{aligned}
$$

A concentration deviation off the stoichiometric composition causes order suppressing to decrease the values $\Omega_{0}$, $K_{0}$ and $S$. It follows therefrom that the most preferable (in terms of detection of the zero-sound mode of collective excitations) is the temperature region that is situated just below the critical temperature $T_{c}$. Explicit form of the temperature dependencies $\Omega_{0}(T), S(T)$ and $K_{0}(T)$ are depicted in Figure 7 where main panel relates to the phase transition of the second order, whereas insertion takes into account polarization effects transforming it into the first order.

According to Eqs. (72) - (74) the diagonal components of complete vertex function have the following hydrodynamic form:

$$
\begin{array}{r}
\Gamma^{\alpha \alpha}\left(\mathbf{K}, \Omega_{S}\right)=2 \pi \chi \frac{\Omega_{S}+\frac{1}{2} \Omega_{0}}{\Omega_{S}^{2}+\Omega_{0} D \mathbf{K}^{2}} \\
\Omega_{S} \equiv 2 \pi S T, \quad S=0, \pm 1, \ldots
\end{array}
$$

Then, taking into account the sum rule [10] (cf. Eqs. (35))

$$
T \sum_{S=-\infty}^{\infty} \frac{1}{\Omega_{S}^{2}+\epsilon^{2}}=\frac{1}{2 \epsilon} \operatorname{coth} \frac{\epsilon}{2 T},
$$

for instant vertex function $\Gamma^{\alpha \alpha}(\mathbf{K}) \equiv T \sum_{S} \Gamma^{\alpha \alpha}\left(\mathbf{K}, \Omega_{S}\right)$ we obtain

$$
\Gamma^{\alpha \alpha}(\mathbf{K})=\frac{\pi}{2} \chi \sqrt{\frac{\Omega_{0}}{D \mathbf{K}^{2}}} \operatorname{coth} \frac{\sqrt{\Omega_{0} D \mathbf{K}^{2}}}{2 T} .
$$

In the hydrodynamic regime $K \ll T / \sqrt{\Omega_{0} D}$ this expression arrives at the known Bogolyubov singularity [8]

$$
\Gamma^{\alpha \alpha}(\mathbf{K})=\pi \frac{\chi T}{D \mathbf{K}^{2}}
$$

that keeps this form for off-diagonal components of the vertex function as well.

\section{CONCLUSIONS}

Above consideration shows that within the single excitation representation the study of the solid solution ordering can be achieved by analogy with the microscopic theory of superconductivity [14], 15]. Description of the collective excitations requires their self-consistent consideration along with single ones. The hydrodynamic behavior of the system is presented as the result of interference of the condensate and fluctuation components the last of which is of diffusive type. This interference results in appearance of the reactive mode corresponding to the zero-sound.

It is naturally, the scheme developed allows one to reproduce complete picture of the solid solution ordering within mean-field approximation [1] - [6]. Setting aside well-known information along this approach, let us focus on main results of our consideration that are not achieved within mean-field scheme.

First, we have found the temperature dependence of the long-range order parameter for different differences of the chemical potentials of the components $\mu \equiv \mu_{A}-\mu_{B}$ accounted from Fermion energy $\varepsilon$ (see Figure 2). Taking the latter as the origin, we obtain a scope of ordering solid solutions is determined by the condition

$$
\mu_{A}-\mu_{B}<\Delta_{0} \equiv W / 2
$$

fixing degree of the chemical affinity of the components with respect to the characteristic value $W \equiv-\sum_{m} w_{l m}$ of mixing energy (6). In accordance with Eq. (40), it means the difference of sublattice concentrations $2 \widetilde{C} \equiv$ $C_{1}-C_{0}$ could not take magnitudes more than one.

The second of practical results allows us to determine the boundary composition of the ordering phase $A B_{n}$ :

$$
\bar{C}=\frac{\sqrt{\bar{m}(1-\bar{m})}-\bar{m}}{(n+1)(1-2 \bar{m})}, \quad \bar{m} \equiv \frac{\mu_{A}-\mu_{B}}{\Delta_{0}} .
$$

As distinct from equality (60) related to the simplest phase $A B$, here we consider the concentration domain $0 \leq \bar{C} \leq(n+1)^{-1}$ instead of $0.5 \leq \bar{C} \leq 1$. According to relation (87), in perfectly ordering solution where $\mu_{A}=\mu_{B}$, the solubility limit is $\bar{C}=0$ and grows anomalously fast with decreasing degree of the chemical affinity of the components with respect to the mixing energy. Moreover, if we insert impurities or defects with concentration $x \ll 1$, the parameter of external influence varies as

$$
\bar{m}=m_{0}+\beta x
$$

where the value $m_{0}$ is inherent in the proper solution $A B_{n}, \beta$ is constant taking both positive and negative signs. Thus, alloy doping arrives at lowering boundary concentration in the case $\beta<0$ and its growth otherwise.

Principle advantage of our approach is a possibility to study solid solutions with arbitrary composition to elaborate complete thermodynamic picture of the long-range ordering process. According to Figures 3, 4 the order parameter decays monotonically with increase of both temperature and concentration, whereas the sublattice difference of the chemical potentials suffers abruption with maximal value $\tilde{\mu} \sim \Delta_{0} / 2$ at phase transition related to intermediate compositions. This allows us to estimate the surface tension coefficient $\sigma \sim \widetilde{\mu} \xi / v$ as follows:

$$
\sigma=c \frac{W \xi}{v}, \quad c \sim 0.1
$$

where $\xi, v$ are characteristic values of the correlation length and the atom volume, respectively. Another physical result is that the second order phase transition is 
transformed into the first one with passing out of the compositional domain $0.318<\bar{C}<0.682$. However, a difference between these transitions is so weak to be observed in Figure $3 \mathrm{a}$.

Much more important is the influence of collective excitations whose polarization effects transform the phase transition to the first order always (see the first of Eqs. (65)). Related collective mode is of a reactive nature in the long-wave region limited by the wave number (74). The dispersion law (75) of this mode can be observed as the Zener peak of the internal friction. According to Figure 7 proper frequency and boundary wave number of this peak decay monotonically with temperature increase, whereas characteristic velocity has a maximum at intermediate temperatures in ordering domain. A deviation off the stoichiometric composition causes suppress- ing of the above zero-sound mode to be pronounced in the temperature region just below the critical temperature. It is worthwhile to notice the polarization effects are relevant to the static (condensate) component of Green function (72) related to frequency $\Omega=0$, whereas the Goldstone mode of the symmetry restoration is represented by the instant vertex function (85) related to time $t=0$.

\section{Acknowledgments}

In this work, financial support by STCU, project 1976, is gratefully acknowledged.
[1] A.D. Bruce, R.A. Cowley, Structural Phase Transitions (Taylor and Francis Ltd., London, 1981).

[2] M.A. Krivoglaz, The Theory of X-Ray and Thermal Neutron Scattering From Real Crystals (Plenum, New York, 1969); Diffuse Scattering of X-rays and Thermal Neutrons by Fluctuational Inhomogeneities of Imperfect Crystals (Springer, Berlin, 1996).

[3] D. de Fontaine, in: Phenomena in Alloys, Magnets, and Superconductors, ed. R.E. Millis (McGraw-Hill, New York, 1971); Solid State Physics 34, 73 (1979).

[4] A.G. Khachaturyan, Theory of Structural Transformations in Solids (Wiley, New York, 1983).

[5] A.A. Katsnelson, A.I. Olemskoi, Microscopic Theory of Nonhomogeneous Structures, (Mir Publishers, Moscow, 1990).

[6] F. Ducastelle, Order and Phase Stability in Alloys (Elsevier, New York, 1991).
[7] J. Zinn-Justin, Quantum Field Theory and Critical Phenomena (Clarendon Press, Oxford, 1993).

[8] N.N. Bogolyubov, Quasi-mean Values in the Problems of Statistical Mechanics. In Coll. Statist. Phys. and Quantum Theory of Field (Nauka, Moscow, 1973).

[9] L.D. Landau, E.M. Lifshits, Course of Theoretical Physics (Pergamon, Oxford, 1980), Vol. 5.

[10] A.P. Prudnikov, Yu.A. Brychkov, O.I. Marichev, Integrals and Series (Nauka, Moscow, 1981).

[11] D. Vollhardt, P. Wölfle, Phys. Rev. B22, 4666 (1980).

[12] T. Kopp, J. Phys. C17, 1897, 1919 (1984).

[13] V.S. Postnikov, Internal Friction in Metals (Metallurgiya, Moscow, 1974).

[14] G. Shriffer, Theory of Superconductivity (Nauka, Moscow, 1979).

[15] R. Haussmann, Z. Phys. B91, 291 (1993). 
a) $\quad-\boldsymbol{G}=$

b)

$-\boldsymbol{G}^{(0)}=$

c)

${ }_{m}^{l} \perp_{m}^{l}=-\mathcal{W}_{l m}$

d)

$=-\hat{\Gamma}$

e)

$=$

f)

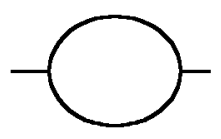

$=-\hat{\Sigma}$

g)

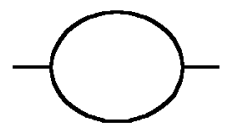

$=$

h)

$=\hat{\Pi}$

i)

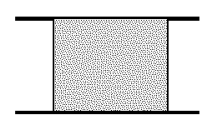

$=I+$

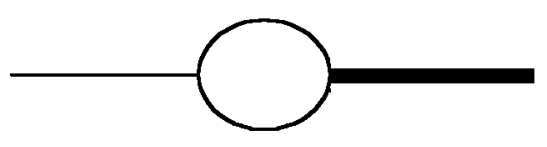




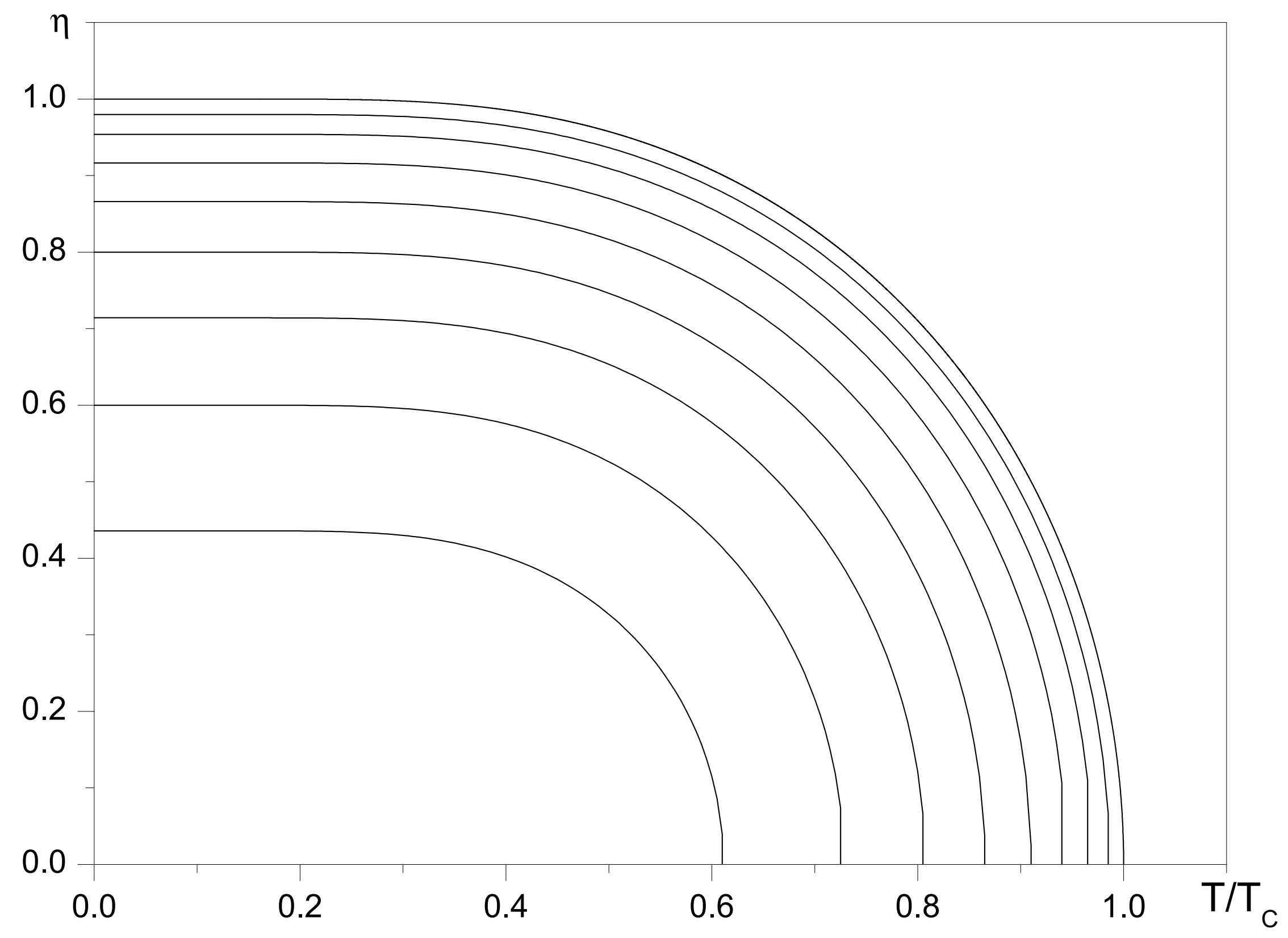



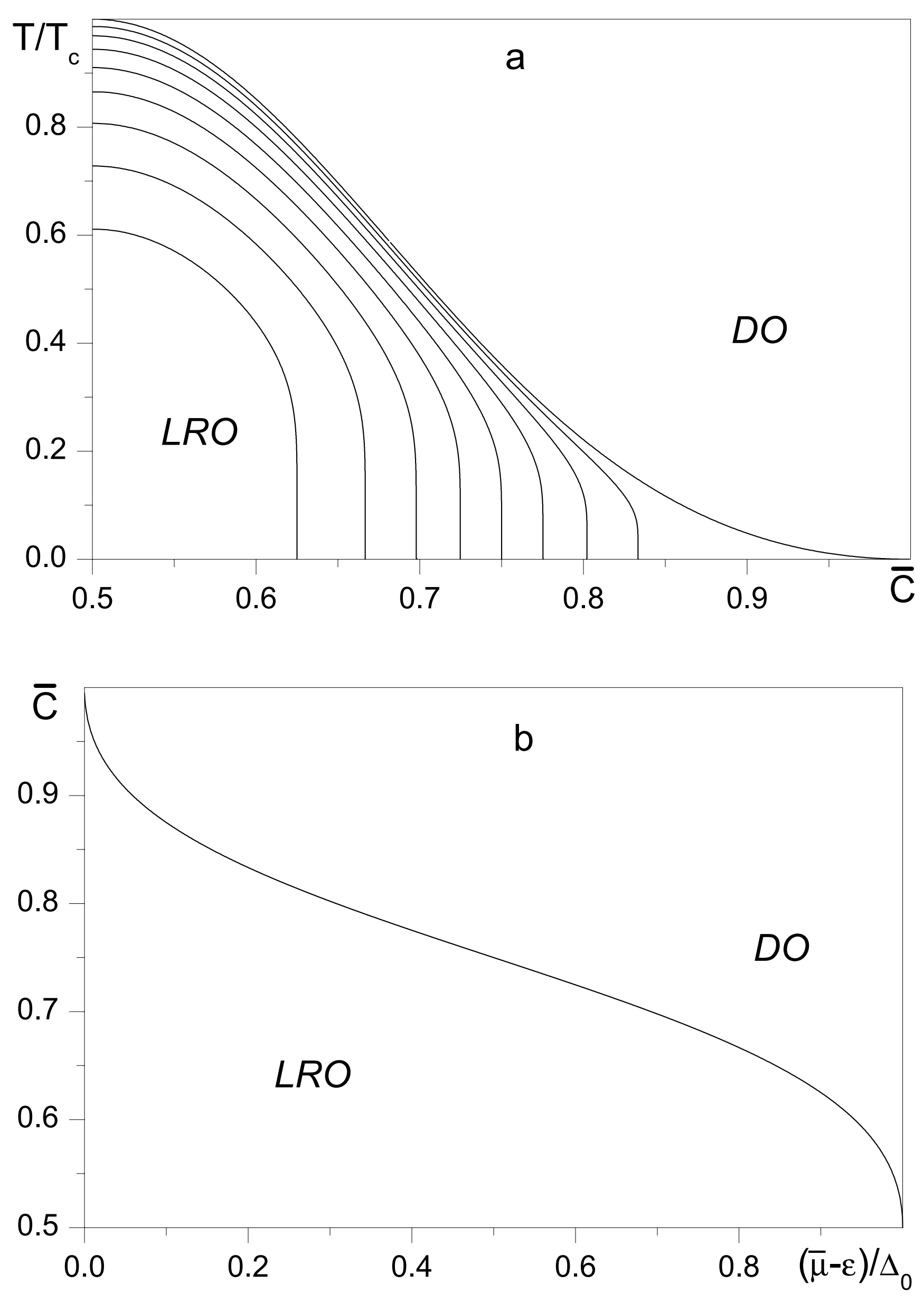


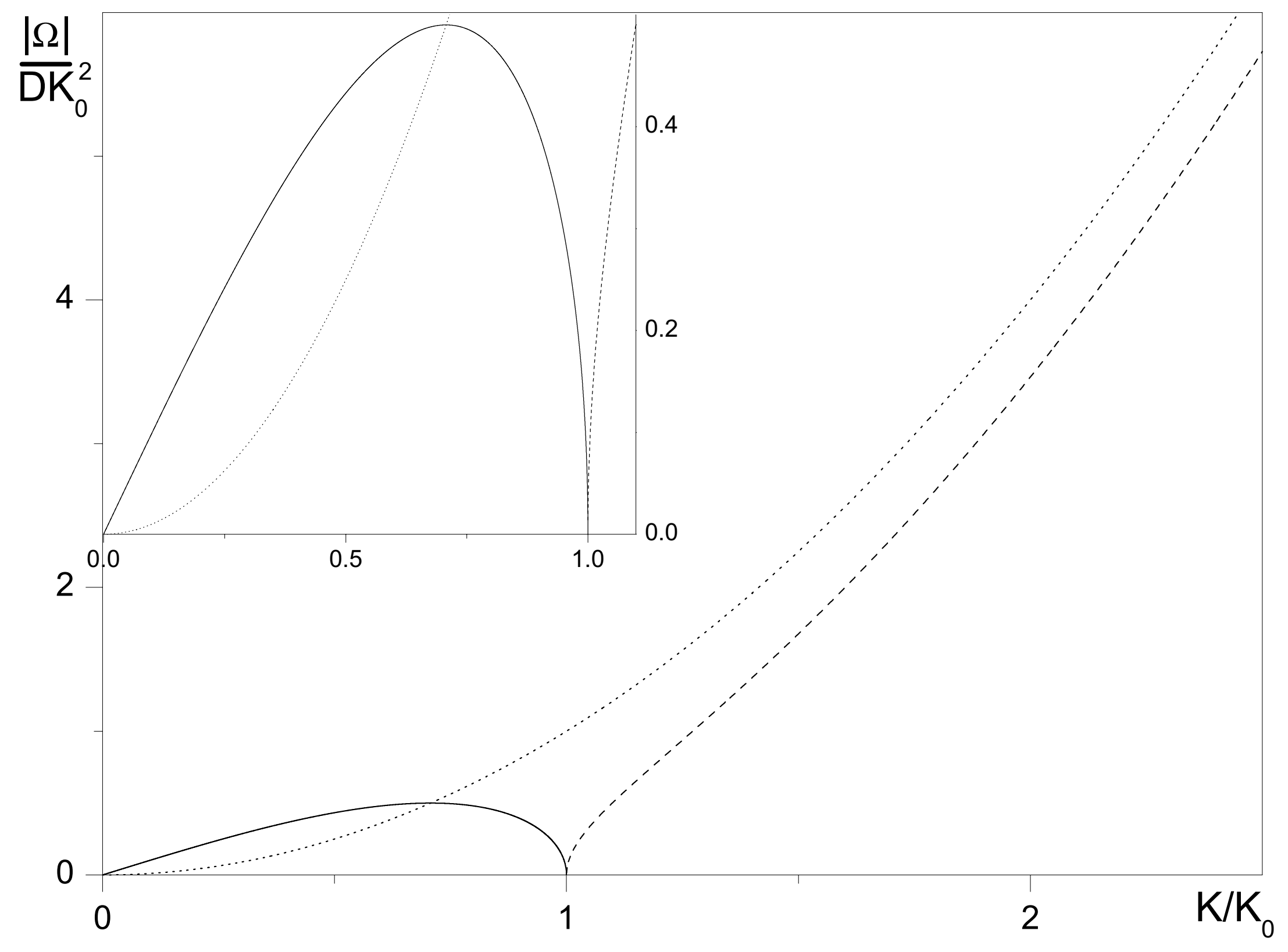




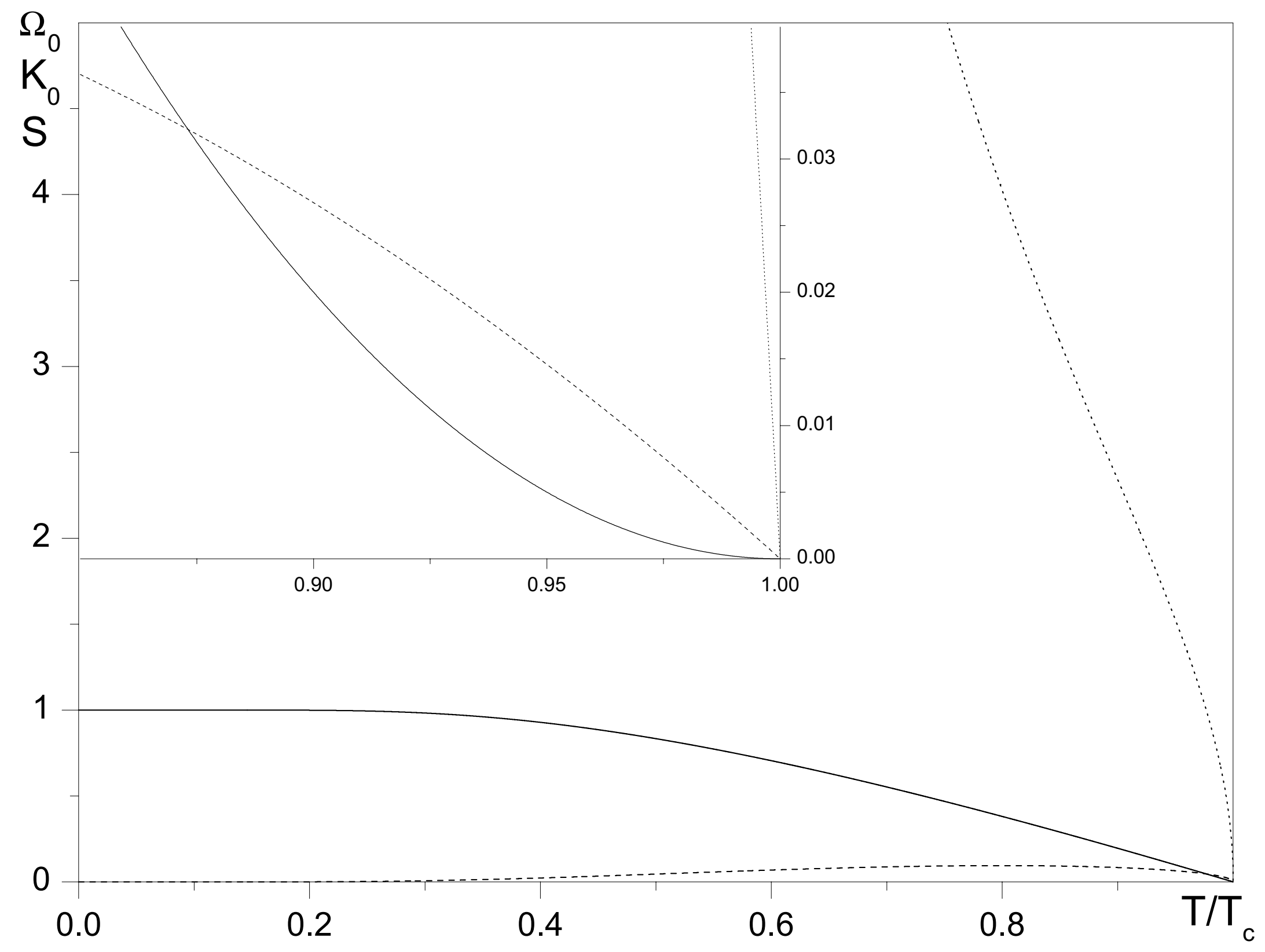

